\title{
Elder Self-Neglect Is Associated with an Increased Rate of 30-Day Hospital Readmission: Findings from the Chicago Health and Aging Project
}

\author{
XinQi Dong ${ }^{a}$ Melissa A. Simon ${ }^{b}$

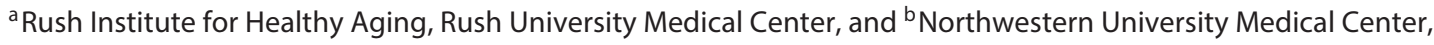 \\ Chicago, III., USA
}

\begin{abstract}
Key Words
Elder self-neglect · 30-Day hospital readmission .

Population-based study
\end{abstract}

\begin{abstract}
Background/Aim: Elder self-neglect is associated with increased morbidity and mortality. The objective of this study is to examine the prospective relationship between reported elder self-neglect and the rate of 30-day hospital readmission in a community population. Methods: We conducted a prospective population-based study of community-dwelling older adults who participated in the Chicago Health and Aging Project. Of the 7,219 participants in the Chicago Health and Aging Project, a subset of 1,228 participants was reported to the social services agency for suspected elder self-neglect. The primary predictor was elder self-neglect reported to the social services agency. The outcome of interest was the annual rate of 30-day hospital readmission calculated from the Center for Medicare and Medicaid System hospitalization data from 1993 to 2009. Poisson regression models were used to assess these relationships. Results: The average annual rate of 30-day hospital readmission for those without elder self-neglect was 0.2 (SD 0.7) and for those with reported elder self-neglect 0.9 (SD 2.8). After adjusting for sociodemographic and socioeconomic characteristics, medical comorbidities, cognitive function, physical function, and psychosocial well-being, elders who self-neglect had a sig-
\end{abstract}

nificantly higher rate of 30-day hospital readmission (rate ratio $2.50,95 \%$ confidence interval 2.02-3.10). Greater self-neglect severity [mild: parameter estimate (PE) 1.09, standard error (SE) 0.19, $p<0.001$; moderate: PE 0.84, SE 0.13, $p<$ 0.001 ; severe: PE 1.24, SE 0.40, $p=0.002$ ] was associated with increased annual rates of 30-day hospital readmission after considering the same confounders. Interaction term analyses suggest that the significant relationship between selfneglect and 30-day hospital readmission was not moderated by medical conditions, cognitive impairment, physical disability, or psychosocial well-being. Conclusion: Reported elder self-neglect was associated with increased rates of 30day hospital readmission in this community population. Greater self-neglect severity was associated with a greater increase in the rate of 30-day hospital readmission.

(c) 2014 S. Karger AG, Basel

\section{Introduction}

Elder self-neglect is an understudied public health issue and is associated with significant adverse health outcomes. Although there is a great paucity in our current understanding of self-neglect, a recent publication from the Chicago Health and Aging Project (CHAP) suggests that 1 out of 9 older adults experiences some form of selfneglect in a community setting [1], and evidence suggests that reports of self-neglect to social services agencies are

\section{KARGER 125}

(c) 2014 S. Karger AG, Basel

0304-324X/14/0611-0041\$39.50/0

E-Mail karger@karger.com

www.karger.com/ger
XinQi Dong, MD, MPH

Rush Institute for Healthy Aging, Rush University Medical Center

1645 West Jackson, Suite 675

Chicago, IL 60612 (USA)

E-Mail xinqi_dong@rush.edu 
on the increase [2]. Moreover, elder self-neglect is independently associated with an increased risk of premature all-cause and cause-specific mortality, and there is a gradient association between higher self-neglect severity and greater risk for all-cause mortality $[3,4]$. Furthermore, self-neglect has great significance not only to healthcare providers and local and state social services agencies, but also to public health advocates, legal profession, community-based groups, and other relevant aging disciplines.

The National Centers on Elder Abuse categorizes elder self-neglect '... as the behavior of an elderly person that threatens his/her own health and safety. Self-neglect generally manifests itself in an older person as a refusal or failure to provide himself/herself with adequate food, water, clothing, shelter, personal hygiene, medication (when indicated), and safety precautions' [5]. Recent epidemiological research has led to significant improved understanding of the self-neglect and its specific subtypes in community-dwelling aging populations $[6,7]$. Equally important, these self-neglecting behaviors could potentially lead to increased utilization of healthcare services, which will likely place an added burden on the current healthcare systems $[8,9]$. However, there remain gaps in our understanding in the health services utilizations among those who self-neglect.

Hospitalization is one of the greatest contributors of rising cost in our current healthcare system. Over the last century, there have been remarkable scientific advances and increased ability to devise preventive health services and disease prevention strategies in order to prevent unnecessary hospitalizations. Prior case reports suggest frequent health services utilization among those identified as self-neglectors $[10,11]$. Recent epidemiological studies have provided conflicting results in health services utilizations among self-neglectors after being identified by the social services agency $[8,9,12,13]$.

Preventable hospital readmission is a significant avoidable cost in the US healthcare system, costing an estimated USD 25 billion annually [14]. Nearly 1 in every 5 Medicare patients discharged from the hospital is readmitted within 30 days [15]. This is of particular concern as the Affordable Care Act is taking effect and Medicare is concentrating on reducing hospital readmission by $20 \%$ or on potentially imposing financial penalties on hospitals with higher than average readmission rates. A recent study indicates that self-neglect is independently associated with an increased rate of hospitalization [9], yet the association between self-neglect and 30-day hospital readmission remain unclear.
Moreover, most prior epidemiological research has categorized self-neglect dichotomously as 'self-neglect' or 'no self-neglect'. However, self-neglect, like many other geriatric syndromes, occurs along a continuum of severity, rather than in two discrete categories [16]. Evidence suggests that improved understanding of the full spectrum of self-neglect is just as important, and that there is a gradient association between greater self-neglect severity and morbidity and mortality $[3,4]$. However, we are not aware of any study that has systematically quantified the relations between the spectrum of self-neglect severity and 30-day hospital readmission in community-dwelling populations.

In this report, we aim to quantify (1) the relationship between reported self-neglect and the rate of 30-day hospital readmission within a prospective population-based study, and (2) the relationship between the continuum of self-neglect severity and 30-day hospital readmission in the same population. We hypothesized that older adults with reported self-neglect have increased rates of 30-day hospital readmission, and that there is a gradient relationship between greater self-neglect severity and a higher rate of 30-day hospital readmission. Addressing these gaps will contribute to the current paucity in our understanding of the consequences of self-neglect, will be critical to educate the public on the consequences of self-neglect and provide data that could inform practice and policy at the local, state and national level.

\section{Methods}

\section{Setting}

CHAP, initiated in 1993, aimed to examine risk factors for Alzheimer's disease. Its participants include residents of three adjacent neighborhoods on the south side of Chicago. More indepth details of the study design of CHAP have been previously published $[17,18]$. Briefly, the study enrolled residents aged 65 years and older of three adjacent neighborhoods on the south side of Chicago. In 1993, the study began with a complete census of the community area. The census identified 7,813 age-eligible residents, 6,158 (78.9\%) of whom were enrolled between 1993 and 1997. In 2000, CHAP began to enroll successive cohorts of participants from the study community who had turned 65 since inception of the study. Data collection occurs in 3-year cycles, with each follow-up cycle beginning after the conclusion of the previous cycle. The follow-up participation rate averaged $80-$ $85 \%$ of survivors at each cycle. Each data collection cycle includes an in-person interview conducted in the participants' homes. The interviews include standardized questionnaires and tests for the assessment of health history and environmental observations.
Dong/Simon 


\section{Participants}

This study included participants who were enrolled in the CHAP study and had data on hospitalization history $(n=7,219)$ obtained from the Centers for Medicare and Medicaid Services (CMS). From this cohort, we identified 1,228 participants who were reported to the social services agency for suspected elder selfneglect. These 1,228 participants were a subset of the total cohort of 7,219 participants. Suspected cases of elder self-neglect were reported by friends, neighbors, family, social workers, city workers, healthcare professionals, and others. The reports were usually initiated based on the concerns for the health and safety for the older adult, which in turn would initiate a wide array of social and legal services to help them. CHAP and social services data were matched using variables of date of birth, gender, race, home telephone number and exact home address. All CHAP participants received structured, standardized in-person interviews that included assessment of health history. Written informed consent was obtained and the study was approved by the Institutional Review Board at Rush University Medical Center.

\section{Predictor: Reported and Confirmed Elder Self-Neglect}

Elder self-neglect in this study was based on all suspected cases reported to a social services agency. When a case was reported, a case worker performed a home assessment, which rates the unmet needs in the domains of personal hygiene and grooming, household and environmental hazards, health needs and overall home safety concerns. A total of 15 items were used to rate the degree of unmet needs, and each item was scored on a scale of $0-3$, with the higher number indicating greater danger to health and safety. The level of severity was rated by case workers based on their concerns for the client's personal health and safety, with the maximum cumulative score of 45 .

Confirmed self-neglect in this study was defined as anyone with a score of 1 or greater $(n=963)$ which are a subset of the reported cases of self-neglect to the social services agency. Through this approach it will permit a broader examination of elder self-neglect severity across the continuum. Elder self-neglect severity (only among those with confirmed elder self-neglect) refers to the scores 1-45, with higher scores within this range indicating greater levels of elder self-neglect severity. The details of this measure have been previously described [19-24]. Available information from the social services agency internal report [25] showed that this measure had inter-rater reliability coefficients $>0.70$ and the internal consistencies were high with Cronbach's $\alpha$ of 0.95 [21]. In addition, the external validity of the measure was assessed and shown to predict an increased risk of premature mortality [3].

\section{Outcome: Hospital Readmission}

Hospitalization records were abstracted from the Medicare Standard Analytic Files (SAFs) obtained from CMS. CMS has approved the Study Protocol and Data Use Agreement with the CHAP study to obtain CMS data. The CHAP study has successfully linked participants and their CMS claims data for the Medical Denominator Files and the SAFs which contain the records of hospitalization. 30-day hospital readmissions were characterized by two consecutive hospital admissions, where the date of discharge from the first hospitalization and the date of admission from the second hospitalization is $\leq 30$ days for a given participant. For each participant, we have abstracted and summarized SAFs on their number of 30-day hospital readmissions after the identification of self-neglect to the last available CMS data in December 2009

\section{Covariates}

Demographic variables include age (in years), gender (men or women), race (self-reported: non-Hispanic Black vs. non-Hispanic White), education (years of education completed), and income categories ( 1 = USD 0-4,999; 2 = USD 5,000-9,999; $3=$ USD 10,000-14,999; 4 = USD 15,000-19,999; 5 = USD 20,000-24,999; $6=$ USD 25,000-29,999; 7 = USD 30,000-34,999; 8 = USD 35,00049,999; 9 = USD 50,000-74,999; $10=$ USD $\geq 75,000)$. The parent CHAP study also collected self-reported medical conditions of hypertension, diabetes mellitus, stroke, coronary artery disease, hip fracture, and cancer.

A battery of four cognitive function tests was administered: the Mini-Mental State Examination (MMSE) [26], immediate and delayed recall of brief stories in the East Boston Memory Test [27] and the Symbol Digit Modalities Test [28]. To assess global cognitive function with minimal floor and ceiling artifacts, we constructed a summary measure for global cognition based on all four tests. Individual test scores were summarized by first transforming a person's score on each individual test to a z-score, which was based on the mean and standard deviation (SD) of the distribution of the scores of all participants on that test, and then averaging $\mathrm{z}$ scores across tests to yield a composite score for global cognitive function. This procedure has the advantage of increasing power by reducing random variability present within tests, as well as reducing floor and ceiling effects of particular tests. In addition, it produces a composite score that is approximately normally distributed.

Physical function was assessed using the Katz Index of Activities of Daily Living (Katz ADL), which measured limitations in an individual's ability to perform basic self-care tasks [29]. Physical function was also assessed by direct performance testing, which provided a comprehensive objective and detailed assessment of certain abilities. Lower extremity performance tests consisted of measures of tandem stand, timed walk, tandem walk, and the ability to rise to a standing position from a chair. The tests requiring walking performance were quantified in terms of the number of seconds to complete the task. Other tests were measured in terms of the number of trials completed within a specified time period. Most of these performance tests were used in the Established Populations for Epidemiologic Studies of the Elderly (EPESE) project [30] and in other large-scale studies of disability. Summary measures of the above tests were created as physical performance test scores. Lower scores indicate impairment in the above activities and tasks which are often needed for independent living and may contribute toward physical disability.

Psychosocial factors have been associated with an increased risk for elder abuse [31-35]. Psychosocial factors included assessment of depressive symptoms, social network and social engagement. Symptoms of depression were measured using a modified version [36] of the Center for Epidemiologic Studies of Depression Scale (CES-D) [37]. The social network was summarized as the total number of children, relatives and friends seen at least monthly [38]. Social engagement was assessed by asking how often older adults participate in social activities such as going to movies, library, senior centers and other social events outside of the house. The scale consists of 12 items and a 4-point scale of frequency and is summarized for this study. 


\section{Analytic Approach}

Univariate analyses were provided for those with and without 30-day hospital readmission groups across age, gender, race, education, income, medical comorbidities, and cognitive function, physical function, depressive symptoms, and social engagement. Our independent variables of interest were reported self-neglect, confirmed self-neglect and self-neglect severity (only derived from confirmed self-neglect). Our outcome of interest was an annual rate of 30-day hospital readmission. In addition, we calculated the annual rate of 30-day hospital readmission for the different severities of elder self-neglect (mild $=$ score of $1-15$; moderate $=$ score of 16-30; and severe $=$ score of 31-45). We compared the differences in the rate of 30-day hospital readmission between groups.

Poisson regression models were used to quantify the relation between elder self-neglect and the rate of 30-day hospital readmission. Given the participants were recruited at different time points throughout the CHAP study, log (time) variables were used in all models. We used a series of models to consider these relationships, taking into consideration the potential confounders. In our core model (model A), we included age and sex. In addition, we added to the prior model the variables of race, education and income to quantify the association between elder abuse and 30-day hospital readmission outcomes (model B). Moreover, we added to the prior model common medical comorbidities of hypertension, coronary artery disease, stroke, hip fracture, cancer, and diabetes as well as levels of cognitive function and physical function (model C). Finally, models were repeated controlling for additional psychosocial measures (model D). We also repeated the prior models $\mathrm{A}-\mathrm{D}$ to examine the association between elder self-neglect and the rate of 30-day hospital readmission. We then examined the relationship between the full spectrum of elder self-neglect severity as a continuous variable and the rate of 30-day hospital readmission by repeating models $\mathrm{A}-\mathrm{D}$. Moreover, we repeated these models for levels of self-neglect severity (mild $=1-15$; moderate $=16-30$; severe $=31-45$ ) and the rate of 30-day hospital readmission. Lastly, we conducted interaction term analyses for the same models to examine the effect of health-related factors and self-neglect, with respect to the rate of 30-day hospital readmission (i.e. medical conditions $\times$ self-neglect, cognitive function $\times$ self-neglect, physical function $\times$ self-neglect, depressive symptoms $\times$ self-neglect and social engagement $\times$ self-neglect). Rate ratio (RR), 95\% confidence interval (CI), standardized parameter estimates (PE), standard error (SE), and $\mathrm{p}$ values were reported for the regression models. Analyses were carried out using SAS ${ }^{\circledR}$ version 9.2 (SAS Institute, Inc., Cary, N.C., USA).

\section{Results}

\section{Baseline Characteristics}

Of the 7,219 CHAP participants in this study, a subset of 1,998 participants had 30-day hospital readmission and 5,221 participants did not have 30-day hospital readmission. The mean age of those with 30-day readmission was 75.0 years $(S D=7.1)$ and of those without 73.2 years (7.2) (table 1). The annual rate of 30 -day hospital readmission for those without self-neglect was $0.18(0.72)$ and
Table 1. Characteristics of the cohort by 30 -day hospital readmission status $(\mathrm{n}=7,219)$

\begin{tabular}{lcc}
\hline & Readmission & \\
\cline { 2 - 3 } & yes $(\mathrm{n}=1,998)$ & no $(\mathrm{n}=5,221)$ \\
\hline Age, years & $75.0 \pm 7.1$ & $73.2 \pm 7.2$ \\
Male & $867(43.4)$ & $2,093(40.1)$ \\
Black & $1,185(59.3)$ & $3,260(62.4)$ \\
Education, years & $11.9 \pm 3.7$ & $12.4 \pm 3.5$ \\
Income categories & $4.9 \pm 2.5$ & $5.3 \pm 2.5$ \\
Medical conditions & $1.2(1.0)$ & $1.0(0.9)$ \\
$\quad$ Coronary artery disease & $380(19.1)$ & $672(12.9)$ \\
$\quad$ Stroke & $278(13.9)$ & $482(9.2)$ \\
$\quad$ Cancer & $411(20.6)$ & $984(18.9)$ \\
$\quad$ Hypertension & $1,061(53.3)$ & $2,614(50.3)$ \\
Diabetes & $222(11.1)$ & $354(6.8)$ \\
Hip fracture & $93(4.7)$ & $171(3.3)$ \\
Global cognitive function & $0.0 \pm 0.9$ & $0.2 \pm 0.8$ \\
MMSE & $25.2 \pm 6.2$ & $26.1 \pm 5.1$ \\
Katz ADL & $0.6 \pm 1.4$ & $0.4 \pm 1.2$ \\
Physical performance testing & $9.1 \pm 4.3$ & $10.3 \pm 3.8$ \\
Depressive symptoms & $1.8 \pm 2.2$ & $1.5 \pm 1.9$ \\
Social engagement & $2.1 \pm 1.7$ & $2.4 \pm 1.7$ \\
Elder self-neglect & $390(31.8)$ & $1,608(26.8)$ \\
\hline
\end{tabular}

Values are presented as $\mathrm{n}(\%)$ or mean $\pm \mathrm{SD}$.

Table 2. Elder self-neglect and annual rate of 30-day hospital readmission

\begin{tabular}{llll}
\hline & Mean \pm SD & Z test & p value \\
\hline No reported self-neglect $(\mathrm{n}=5,991)$ & $0.18 \pm 0.72$ & & \\
Reported self-neglect $(\mathrm{n}=1,228)$ & $0.89 \pm 2.77$ & 6.63 & $<0.001$ \\
Confirmed self-neglect $(\mathrm{n}=963)$ & $0.96 \pm 2.88$ & 7.02 & $<0.001$ \\
Self-neglect severity & & & \\
$\quad$ Mild $(\mathrm{n}=227)$ & $0.97 \pm 3.31$ & 50.43 & $<0.001$ \\
$\quad$ Moderate $(\mathrm{n}=673)$ & $0.89 \pm 2.55$ & & \\
$\quad$ Severe $(\mathrm{n}=63)$ & $1.74 \pm 4.18$ & & \\
\hline
\end{tabular}

for those with reported elder self-neglect 0.89 (2.77; p < 0.001 ) (table 2). Similar results were found for confirmed self-neglect. In addition, the study found an increase in the levels of self-neglect severity and 30-day hospital readmission. The annual rate of hospitalization utilization for mild self-neglect was 0.97 (3.31), for moderate selfneglect 0.89 (2.55), and for severe self-neglect 1.74 (4.18; $\mathrm{p}<0.001)$.
Dong/Simon 
Table 3. Elder self-neglect and the rate of 30-day hospital readmission

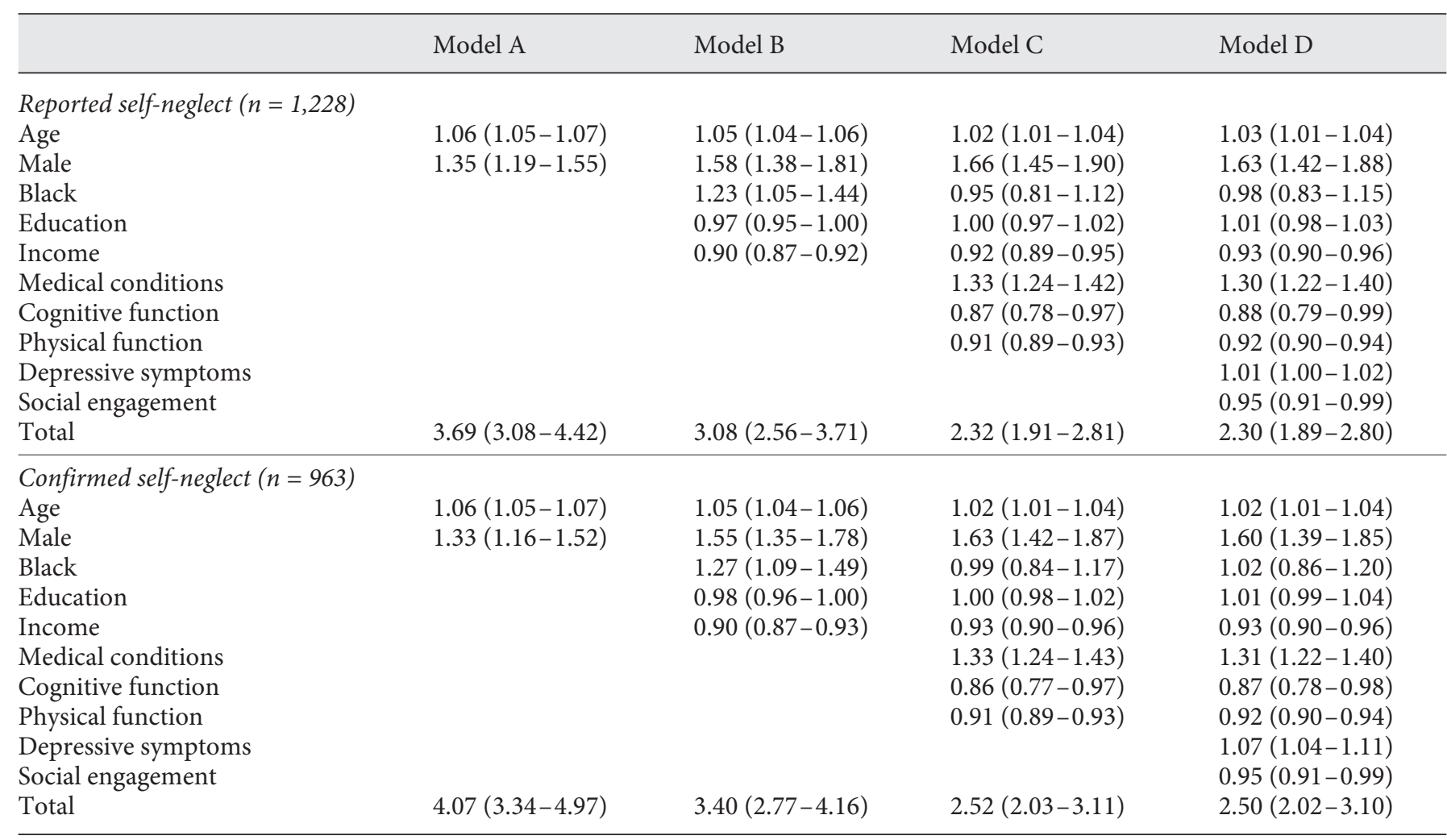

Results expressed as RR and 95\% CI (in parentheses) for the 30-day readmission outcome.

\section{Elder Self-Neglect and Rate of 30-Day Hospital Readmission}

In the initial Poisson regression model adjusting for age and gender, we found that reported elder self-neglect independently predicted the increased rate of 30-day hospital readmission (RR 3.69, 95\% CI 3.08-4.42) (model A, table 3). After adding socioeconomic variables of race, education and income as potential confounders (model B), the association diminished slightly (RR 3.08, 95\% CI 2.56-3.71). We then added common chronic medical conditions of hypertension, diabetes, stroke, cancer, thyroid disease, myocardial infarction, cognitive function, and physical function to the model (model C), and the association decreased but remained significant (RR 2.32, 95\% CI 1.91-2.81). The last model (model D), after adjusting for depressive symptoms and social engagement measures, reported that elder self-neglect remained an independent predictor of an increased rate of 30-day hospital readmission (RR 2.30, 95\% CI 1.89-2.80). For confirmed elder self-neglect, the associations were similar (table 3).

Elder Self-Neglect and 30-Day

Readmission

\section{Elder Self-Neglect Severity and 30-Day Hospital Readmission}

To quantify the relation between elder self-neglect severity and 30-day hospital readmission, an initial Poisson regression model adjusting for age and gender was created with 30-day hospital readmission as the outcome (model A). The coefficient representing the association of every point increase in the self-neglect severity score (range 1-45) and 30-day hospital readmission was PE 0.03 , SE 0.01 , RR 1.03, 95\% CI 1.01-1.04, $\mathrm{p}=0.003$, suggesting a statistically significant association between greater severities of elder self-neglect and increased rates of 30-day hospital readmission. After adding for race, income and education (model B), the significant association remains (PE 0.03, SE 0.01, RR 1.03, 95\% CI 1.01$1.04, \mathrm{p}=0.001)$. We then added chronic medical conditions of hypertension, diabetes, stroke, cancer, thyroid disease, coronary artery disease, cognitive and physical function to the model (model C), and the association remained statistically significant (PE 0.02 , SE 0.01 , RR 1.02, $95 \%$ CI 1.01-1.04, $\mathrm{p}<0.01$ ). In the last model (model D), 


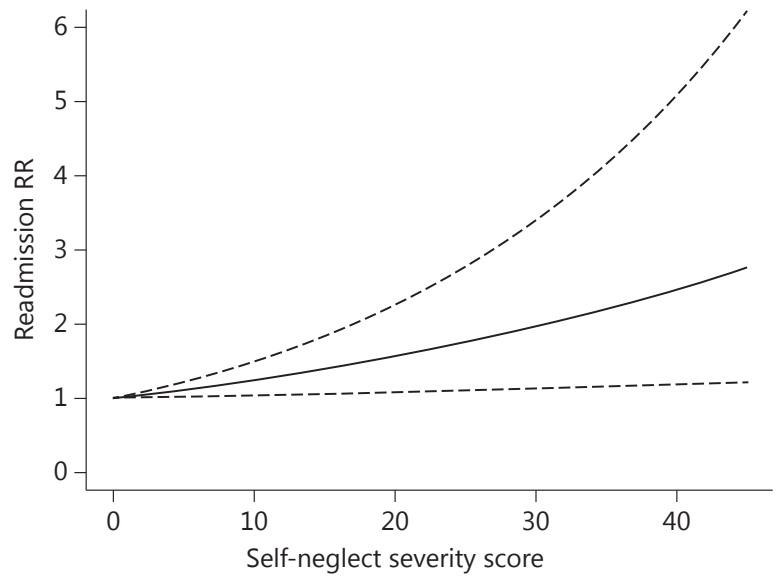

Fig. 1. Elder self-neglect severity and the rate of 30-day hospital readmission. The figure suggests that there is a gradient association between the greater elder self-neglect severity score and the rate of 30-day hospital readmission for 963 self-neglecting older adults. The solid line indicates the readmission RR as self-neglect severity increases. The dashed lines indicate the $95 \%$ CI of the readmission RR. The $\mathrm{x}$-axis represents the elder self-neglect severity score from 1 to 45 and the $y$-axis represents the increase in the rate of 30-day hospital readmission calculated based on the number of 30-day readmissions per year.

Table 4. Elder self-neglect severity and the rate of 30-day hospital readmission

\begin{tabular}{lllllll}
\hline & Models & PE & SE & RR & $95 \%$ CI & p value \\
\hline Mild & A & 1.38 & 0.19 & 4.01 & $2.75-5.84$ & $<0.001$ \\
& B & 1.25 & 0.19 & 3.49 & $2.41-5.07$ & $<0.001$ \\
& C & 1.11 & 0.19 & 3.04 & $2.10-4.42$ & $<0.001$ \\
& D & 1.09 & 0.19 & 3.00 & $2.07-4.34$ & $<0.001$ \\
\hline Moderate & A & 1.37 & 0.12 & 3.94 & $3.12-4.96$ & $<0.001$ \\
& B & 1.18 & 0.12 & 3.27 & $2.50-4.14$ & $<0.001$ \\
& C & 0.84 & 0.13 & 2.32 & $1.82-2.97$ & $<0.001$ \\
& D & 0.84 & 0.13 & 2.33 & $1.81-2.98$ & $<0.001$ \\
\hline Severe & A & 1.88 & 0.38 & 6.55 & $3.06-13.99$ & $<0.001$ \\
& $\mathrm{~B}$ & 1.71 & 0.39 & 5.55 & $2.58-11.94$ & $<0.001$ \\
& C & 1.24 & 0.40 & 3.45 & $1.57-7.58$ & 0.002 \\
& D & 1.19 & 0.41 & 3.30 & $1.48-7.39$ & 0.003
\end{tabular}

Self-neglect severity represents a 1-point increase on the scale of 1-45. Models: (A) adjusted for age, sex, and race; (B) adjusted for $\mathrm{A}+$ education and income; $(\mathrm{C})$ adjusted for $\mathrm{A}+$ hypertension, diabetes, stroke, cancer, hip fracture, coronary artery disease, MMSE, East Boston Memory Test, East Boston Delayed Recall, Symbol Digit Modality Test, and physical function; (D) adjusted for $\mathrm{C}+$ depressive symptoms and social engagement. after adding psychosocial measures to the prior model, the coefficient changed minimally and remained statistically significant (PE 0.02, SE 0.01, RR 1.02, 95\% CI 1.00$1.04, \mathrm{p}<0.01)$. Figure 1 graphically represents greater self-neglect severity (range 1-45) and the rate of 30-day hospital readmission in the fully adjusted model.

We quantified the relation between categorically defined levels of self-neglect severity and the rate of 30-day hospital readmission (table 4). In the core model (model A), mild self-neglect (PE 1.38, SE 0.19, RR 4.01, 95\% CI 2.75-5.84, $\mathrm{p}<0.001$ ), moderate self-neglect (PE 1.37, SE 0.12 , RR 3.94, 95\% CI 3.12-4.96, p < 0.001) and severe self-neglect (PE 1.88, SE 0.38, RR 6.55, 95\% CI 3.06-13.99, $\mathrm{p}<0.001)$ were all independently associated with the increased rate of 30-day hospital readmission. In models B and $\mathrm{C}$, addition of race, education, income, chronic medical conditions, and cognitive function did not significantly change the associations between self-neglect severity and the rate of 30-day hospital readmission. In the fully adjusted model (model D), mild self-neglect (PE 1.09, SE 0.19, RR 3.00, 95\% CI 2.07-4.34, p < 0.001), moderate self-neglect (PE 0.84, SE 0.13, RR 2.33, 95\% CI 1.81$2.98, \mathrm{p}<0.001)$ and severe self-neglect (PE 1.24, SE 0.40, RR 3.45, 95\% CI 1.57-7.58, $\mathrm{p}=0.002$ ) were all independently associated with the increased rate of 30-day hospital readmission.

\section{Interaction Term Analyses between Self-Neglect and Health-Related Factors}

Finally, we examine the interaction effect of self-neglect with health-related factors with respect to the rate of 30-day hospital readmission (table 5). For medical conditions, the interaction term (medical conditions $\times$ self-neglect) was not statistically significant (PE 0.00, SE 0.01, RR 1.00, 95\% CI 0.98-1.02, p = 0.56), suggesting medical conditions did not significantly modify the relationship between self-neglect and the rate of 30-day hospital readmission. Similar non-statistically significant results were found for interaction term analyses for cognitive function, physical function, depressive symptoms, social engagement, and self-neglect with respect to the rate of 30day hospital readmission outcome.

\section{Discussion}

We found that reported and confirmed elder self-neglect independently associated with the increased risk of 30-day hospital readmission. In addition, greater severity of elder self-neglect was associated with a greater risk of
Dong/Simon 
Table 5. Interaction term analyses of self-neglect severity $(n=913)$ with health-related factors and the rate of 30 day hospital readmission

\begin{tabular}{|c|c|c|c|c|c|}
\hline & $\mathrm{PE}$ & SE & $\mathrm{RR}$ & $95 \%$ CI & $\mathrm{p}$ value \\
\hline \multicolumn{6}{|l|}{ Medical conditions } \\
\hline Self-neglect & 0.02 & 0.02 & 1.02 & $0.99-1.05$ & 0.30 \\
\hline Medical conditions & 0.23 & 0.14 & 1.26 & $0.96-1.66$ & 0.10 \\
\hline Self-neglect $\times$ medical conditions & 0.00 & 0.01 & 1.00 & $0.99-1.02$ & 0.56 \\
\hline \multicolumn{6}{|l|}{ Cognitive function } \\
\hline Self-neglect & 0.02 & 0.01 & 1.02 & $1.00-1.04$ & 0.01 \\
\hline Cognitive function & -0.06 & 0.22 & 0.94 & $0.62-1.44$ & 0.79 \\
\hline Self-neglect $\times$ cognitive function & 0.00 & 0.01 & 1.00 & $0.98-1.02$ & 0.69 \\
\hline \multicolumn{6}{|l|}{ Physical function } \\
\hline Self-neglect & 0.01 & 0.02 & 1.01 & $0.97-1.04$ & 0.74 \\
\hline Physical function & -0.06 & 0.04 & 0.94 & $0.87-1.02$ & 0.15 \\
\hline Self-neglect $\times$ physical function & 0.00 & 0.00 & 1.00 & $1.00-1.01$ & 0.19 \\
\hline \multicolumn{6}{|l|}{ Depressive symptoms } \\
\hline Self-neglect & 0.02 & 0.01 & 1.02 & $1.00-1.05$ & 0.06 \\
\hline Depressive symptoms & 0.04 & 0.07 & 1.04 & $0.90-1.19$ & 0.60 \\
\hline Self-neglect $\times$ depressive symptoms & 0.00 & 0.00 & 1.00 & $0.99-1.01$ & 0.97 \\
\hline \multicolumn{6}{|l|}{ Social engagement } \\
\hline Self-neglect & 0.02 & 0.01 & 1.02 & $1.00-1.05$ & 0.10 \\
\hline Social engagement & -0.08 & 0.12 & 0.93 & $0.73-1.18$ & 0.55 \\
\hline Self-neglect $\times$ social engagement & 0.00 & 0.01 & 1.00 & $0.99-1.01$ & 0.89 \\
\hline
\end{tabular}

30-day hospital readmission. Moreover, the significant relationship between self-neglect and 30-day hospital readmission was not moderated through the presence of medical comorbidities, cognitive impairment, physical disability, and psychosocial factors.

A prior study from the EPESE identified 120 cases of self-neglect through manual dataset matching [13] and found that self-neglect was associated with an increased risk of nursing home placement. Another study has also indicated that older adults who have encounters with adult-protective services agencies have greater utilization of behavioral health services [39]. Recent findings from the CHAP study indicate that both reported and confirmed self-neglect has an almost 1.5-fold increase in the rate of hospitalization (RR 1.47, 95\% CI 1.39-1.55) [9]. However, a recent retrospective case-control study of 131 self-neglect cases found no significant differences in healthcare utilizations compared to the matched controls in a Texas population [12].

While there are multiple reasons for the hospital readmission, evidence suggests that improved care transitions and interdisciplinary efforts are critical to potentially reduce hospital readmissions [40-42]. If the self-neglecting behaviors are the seminal factors that lead to the decline in health conditions (congestive heart failure, skin infec- tion, falls, etc.) that warrants hospital admission, then stabilizing the health deteriorations will unlikely change the root of the problem. If a self-neglecting older adult is sent home in whom self-neglecting behaviors are likely to continue, unless healthcare professionals address the issues of self-neglect, it is likely that the vicious cycle will continue and the person's health will deteriorate which may further warrant hospital admission.

Our findings contribute to the field of elder selfneglect and its adverse health outcomes. First, our study systematically examined the prospective association between self-neglect and the rate of 30-day hospital readmissions, demonstrating a significant association between an elder self-neglect increased rate of hospital readmissions. The study population is sociodemographically and socioeconomically diverse and has been well characterized for more than 17 years, which contributes toward the generalizability in community populations of older adults.

Second, our study took into consideration a wide range of potential confounders in the relationship between elder self-neglect and the rate of hospital readmissions. Older age, lower levels of socioeconomic status, more medical comorbidities, lower levels of cognitive and physical health, and psychosocial distress have been as- 
sociated with an increased risk for health services utilization. However, adjusting for these factors did not significantly reduce the significant relationship between selfneglect and the rate of hospital readmissions.

Third, our study examined the continuum of elder self-neglect with respect to the rate of 30-day hospital readmission, rather than strictly categorically defined selfneglect. Our study findings contribute to the associations between self-neglect severity and the rate of hospital readmissions. This information fills an important gap to the better understanding of the potential causal association between self-neglect and health services utilization. It is important for professionals, social services agencies and other relevant disciplines to identify older adults at risk for self-neglect and to intervene before self-neglecting behaviors become more severe, which might then necessitate hospital readmissions.

The potential causal mechanisms between elder selfneglect and hospital readmissions require additional investigation. We took into consideration a series of sociodemographic and socioeconomic characteristics, medical comorbidities, cognitive function, physical function, and psychosocial factors. However, adjustments for these factors did not significantly modify the relationship between self-neglect and the rate of 30-day hospital readmission. Metabolic abnormalities, nutritional deficiencies, infections, injuries, or trauma may be other factors that account for the association between elder self-neglect and the rate of hospital readmissions, but these factors were not considered in this analysis. The severity of medical comorbidities (ejection fraction of a congestive heart failure patient, forced expiratory volume of a chronic obstructive pulmonary disease patient, etc.) could be another important factor in determining the causal mechanisms between self-neglect and hospital readmissions. It is conceivable that self-neglect could exacerbate the existing medical conditions, which could predispose a higher rate of hospital readmissions. Future studies are needed to quantify these relations.

Our study also has limitations. First, using CMS data is a selective underdetection of some services including the use of Veterans Administration facilities and some managed care episodes. This underdetection of our outcomes of interest may potentially underestimate the strength of association between self-neglect and 30-day hospital readmission. Second, this study could not examine the relation between specific indicators/behaviors of self-neglect and the rate of hospital readmissions, as the precise understanding of self-neglect phenotypes (i.e. environmental neglect vs. personal hygiene) could improve our understanding of the causal mechanism. Future studies are needed to examine the relation between specific phenotypes of self-neglect and hospital readmissions. Third, we do not have details on the admitting and discharge diagnosis codes of the first and subsequent hospitalizations to delineate if the readmission diagnoses are the same. Future research is needed to quantify these relationships.

Fourth, our study does not have available data on other health services utilization measures (i.e. outpatient clinic visit, home health visit or nursing home placement). This information could help to explain the temporal relationships between self-neglect and hospital readmissions. Fifth, we do not have information on the client's social support system or the social context of the self-neglecting behaviors that might further increase the risk of hospital readmissions. Sixth, we have neither data on Medicaid status or the duo-eligible status of the participants, nor data on the specific types and reasons for hospital readmissions or the potential differential use of an intensive care unit for those who self-neglect. These data could be important to consider as additional confounders or mediators in our analyses may in part account for the findings in this report. However, this study sets the foundation for future study of self-neglect to systematically examine these issues in representative populations.

Our findings have potential implications in the prevention, detection and management of elder self-neglect. Healthcare professionals should consider routine screening for self-neglect among older patients who may have frequent encounters with healthcare systems. Close monitoring of older adults who self-neglect could help clinicians to more closely monitor the patients and set the basis for future intervention study to examine the effectiveness of screening for self-neglect in the community population.

Our findings could have important implications not only for clinicians, but also across multiple disciplines, which work with older adults with self-neglect. Other relevant medical disciplines, legal professionals, nursing, social workers, social services agencies who work with elders who self-neglect or who are at risk for self-neglect, could be in unique positions to identify and intervene on predisposing factors that might increase the unnecessary hospital readmissions. Our finding could also have implications for legal and law enforcement professionals, especially relating to guardianship proceedings for older adults who have the most severe form of self-neglecting behaviors. Moreover, it is important for aging professionals to mon- 
itor the severity or the progression of self-neglecting behaviors in older adults. Early identification of milder forms of self-neglect and devising targeted prevention and intervention strategies could prevent the deterioration of self-neglect into more severe forms, which in turn could potentially decrease the unnecessary hospital readmissions. Vigorous monitoring and better understanding of factors that might worsen self-neglect severity could also help clinicians to work closely with concerned family members, social workers, legal professionals, health professionals, and public health and community organizations to create a multidisciplinary approach to care for this vulnerable population.

Future epidemiological research is needed to examine the temporal associations of targeted risk/protective factors associated with self-neglect [43]. Future studies are needed to explore the longitudinal association between self-neglect and the rate as well as the intensity of other forms of health services utilization. Future studies are needed to examine the effectiveness of current prevention and intervention strategies for elder self-neglect with respect to health services utilization outcomes in community populations. Future studies are needed to explore the racial/ethnic differences in elder self-neglect and health services utilization in socioeconomically diverse populations [44].

\section{Conclusion}

With the context of a large cohort study, elder selfneglect is independently associated with an increased annual rate of 30-day hospital readmission. In addition, there is an association between greater self-neglect severity and higher rates of hospital readmissions. Future longitudinal research is needed to examine the potential causal inferences between specific self-neglect behaviors and various forms of health services utilization.

\section{Acknowledgments}

We would like to thank the Adult Protective Services staff of Illinois for their continued dedication to protect the vulnerable victims of elder abuse. Dr. Dong is supported by National Institute on Aging grant (R01 AG042318, R01 MD006173, R01 AG11101, and RC4 AG039085), Paul B. Beeson Award in Aging (K23 AG030944), The Starr Foundation, John A. Hartford Foundation, and The Atlantic Philanthropies.

\section{Disclosure Statement}

The authors have no conflicts of interest to disclose.

\section{References}

1 Dong X, Simon MA, Mosqueda L, Evans DA: The prevalence of elder self-neglect in a community-dwelling population: hoarding, hygiene, and environmental hazards. J Aging Health 2012;24:507-524.

2 Teaster PB: A response to abuse of vulnerable adults: the 2000 survey of state Adult Protective Service. 2002. http://www.ncea.aoa.gov/ ncearoot/Main_Site/pdf/research/apsreport030703.pdf.

-3 Dong X, Simon M, Mendes de Leon C, Fulmer T, Beck T, Hebert L, et al: Elder self-neglect and abuse and mortality risk in a communitydwelling population. JAMA 2009;302:517526 .

-4 Dong X, Simon MA, Fulmer T, Mendes de Leon CF, Hebert LE, Beck T, et al: A prospective population-based study of differences in elder self-neglect and mortality between Black and White older adults. J Gerontol A Biol Sci Med Sci 2011;66A:695-704.

5 National Center on Elder Abuse Website. NCEA: the basics. 2006. http://elderabusecenter.org/pdf/research/apsreport030703. pdf.

Elder Self-Neglect and 30-Day

Readmission
6 Dyer CB, Kelly PA, Pavlik VN, Lee J, Doody RS, Regev T, et al: The making of a self-neglect severity scale. J Elder Abuse Negl 2006;18:1323.

7 Dyer CB, Goodwin JS, Pickens-Pace S, Burnett J, Kelly PA: Self-neglect among the elderly: a model based on more than 500 patients seen by a geriatric medicine team. Am J Public Health 2007;97:1671-1676.

8 Dong X, Simon MA, Evans DA: Prospective study of the elder self-neglect and emergency department use in a community population. Am J Emerg Med 2012;30:553-561.

-9 Dong X, Simon MA, Evans D: Elder self-neglect and hospitalization: findings from the Chicago Health and Aging Project. J Am Geriatr Soc 2012;60:202-209.

10 Cybulska E, Rucinski J: Gross self-neglect in old age. Br J Hosp Med 1986;36:21-25.

11 Roe PF: Self-neglect. Age Ageing 1977;6:192194.

12 Franzini L, Dyer CB: Healthcare costs and utilization of vulnerable elderly people reported to Adult Protective Services for self-neglect. J Am Geriatr Soc 2008;56:667-676.
13 Lachs MS, Williams CS, O’Brien S, Pillemer KA: Adult-protective service use and nursing home placement. Gerontologist 2002;42:734739 .

14 Price Waterhouse Cooper Health Research Institute: The Price of Excess: Identifying Waste in Healthcare Spending. New York, Coopers LLP Health Research Institute, 2008.

15 Jencks SF, Williams MV, Coleman EA: Rehospitalizations among patients in the Medicare fee-for-service program. N Engl J Med 2009;360:1418-1428.

16 Dong X, Gorbien M: Decision-making capacity: the core of self-neglect. J Elder Abuse Negl 2005; 17:19-36.

17 Bienias JL, Beckett LA, Bennett DA, Wilson RS, Evans DA: Design of the Chicago Health and Aging Project (CHAP). J Alzheimers Dis 2003;5:349-355.

18 Evans DA, Bennett DA, Wilson RS, Bienias JL, Morris MC, Scherr PA, et al: Incidence of Alzheimer disease in a biracial urban community: relation to apolipoprotein $\mathrm{E}$ allele status. Arch Neurol 2003;60:185-189.

Readmission 
19 Dong X, Mendes de Leon CF, Evans DA: Is greater self-neglect severity associated with lower levels of physical function? J Aging Health 2009;21:596-610.

-20 Dong X, Wilson RS, Simon MA, Rajan B, Evans DA: Cognitive decline and risk of elder self-neglect: the findings from the Chicago Health and Aging Project. J Am Geriatr Soc 2010;58:2292-2299.

21 Dong X, Wilson RS, Mendes de Leon CF, Evans DA: Self-neglect and cognitive function among community-dwelling older persons. Int J Geriatr Psychiatry 2010;25:798-806.

22 Dong X, Simon MA, Evans DA: Characteristics of elder self-neglect in a biracial population: findings from a population-based $\mathrm{co}^{-}$ hort. Gerontology 2010;56:325-334.

23 Dong X, Beck TT, Evans DA: A cross-sectional population-based study of self-neglect and psychosocial factors in a biracial community. Aging Ment Health 2010;14:74-84.

24 Dong X, Simon MA, Wilson RS, Beck TT, McKinnell K, Evans DA: Association of personality traits with elder self-neglect in a community-dwelling population. Am J Geriatr Psychiatry 2011;19:743-751.

25 Illinois Department on Aging: Determination of Need Revision Final Report Volume I. Springfield, Illinois Department on Aging, 1989.

-26 Folstein MF, Folstein SE, McHugh PR: 'Minimental state'. A practical method for grading the cognitive state of patients for the clinician. J Psychiatr Res 1975;12:189-198.

-27 Albert M, Smith LA, Scherr PA, Taylor JO, Evans DA, Funkenstein HH: Use of brief cognitive tests to identify individuals in the community with clinically diagnosed Alzheimer's disease. Int J Neurosci 1991;57:167-178.
28 Smith A: Symbol Digit Modalities Test Manual - Revised. Los Angeles, Western Psychological, 1984.

29 Katz S, Akpom CA: A measure of primary sociobiological functions. Int J Health Serv 1976;6:493-508.

30 Guralnik JM, Simonsick EM, Ferrucci L, Glynn RJ, Berkman LF, Blazer DG, et al: A short physical performance battery assessing lower extremity function: association with self-reported disability and prediction of mortality and nursing home admission. J Gerontol 1994;49:M85-M94.

31 Dong X, Simon MA, Odwazny R, Gorbien $\mathrm{MJ}$ : Depression and elder abuse and neglect among community-dwelling Chinese elderly population. J Elder Abuse Neglect 2008;20: 25-41.

32 Dong X, Beck TT, Simon MA: Loneliness and mistreatment of older Chinese women: does social support matter? J Women Aging 2009; 21:293-302.

33 Dong X, Simon MA: Is greater social support a protective factor against elder mistreatment? Gerontology 2008;54:381-388.

-34 Dong X, Simon MA, Gorbien M, Percak J, Golden R: Loneliness in older Chinese adults: a risk factor for elder mistreatment. J Am Geriatr Soc 2007;55:1831-1835.

35 Dong X, Simon MA: Gender variations in the levels of social support and risk of elder mistreatment in a Chinese Community population. J Appl Gerontol 2010;29:720-739.
36 Kohout FJ, Berkman LF, Evans DA, CornoniHuntley J: Two shorter forms of the CES-D (Center for Epidemiological Studies Depression) depression symptoms index. J Aging Health 1993;5:179-193.

37 Radloff L: The CES-D Scale: a self-report depression scale for research in the general population. Appl Psychol Meas 1977;1:385-401.

38 Cornoni-Huntley J, Brock DB, Ostfeld A, Taylor JO, Wallace RB: Established Populations for Epidemiological Studies of the Elderly Resource Data Book (Rep. No. NIH Publ No. 86-2443). Washington, US Department of Health and Human Services, 1986.

-39 Schonfeld L, Larsen RG, Stiles PG: Behavioral health services utilization among older adults identified within a state abuse hotline database. Gerontologist 2006;46:193-199.

40 Coleman EA, Parry C, Chalmers S, Min SJ: The care transitions intervention: results of a randomized controlled trial. Arch Intern Med 2006;166:1822-1828.

41 Burke RE, Coleman EA: Interventions to decrease hospital readmissions: keys for costeffectiveness. JAMA Intern Med 2013;173: 695-698.

42 Hansen LO, Greenwald JL, Budnitz T, Howell E, Halasyamani L, Maynard G, et al: Project BOOST: Effectiveness of a multihospital effort to reduce rehospitalization. J Hosp Med 2013;8:421-427.

43 Mosqueda L, Dong X: Elder abuse and selfneglect: 'I don't care anything about going to the doctors ... to be honest'. JAMA 2011;306: 532-540.

-44 Dong X, Simon MA: Enhancing national policy and programs to address elder abuse. JAMA 2011;305:2460-2461. 